\title{
Learning Cooperation in a Tangible Moyangsung
}

\author{
Kyoung Shin Park ${ }^{1}$, Hyun-Sang $\mathrm{Cho}^{2}$, Jaewon $\mathrm{Lim}^{3}$, Yongjoo Cho ${ }^{3}$, \\ Seungmook Kang ${ }^{4}$, and Soyon Park ${ }^{4}$ \\ ${ }^{1}$ Multimedia Engineering, Dankook University, \\ San 29 Anseo-dong, Cheonan-si, Chungnam, 330-714, Korea \\ kpark2@hanmail.net \\ ${ }^{2}$ Digital Media Laboratory, Information and Communications University, \\ 517-10 Dogok-dong, Gangnam-gu, Seoul 135-854, Korea \\ ${ }^{3}$ Division of Media Technology, Sangmyung University, \\ 7 Hongji-dong, Jongno-gu, Seoul, 110-743, Korea \\ ${ }^{4}$ Department of Media Art, Jeonju University, \\ 1200 Hyoja-dong, Wansan-gu, Jeonju, 560-759, Korea
}

\begin{abstract}
Tangible Moyangsung is a collaborative virtual heritage environment that employs tangible interfaces to encourage group social interaction and cooperation. It is designed for a group of users to play fortification games for reconstructing a Korean war-defensive castle in virtual reality. This paper describes a historical background and cultural meanings of Moyangsung, and details in design, implementation and a user study of the Tangible Moyangsung system. The study results showed that collaborative work using tangible interfaces helped users to be more engaged in the task and learn cooperation to produce better performance.
\end{abstract}

Keywords: Virtual Heritage Environment, Tangible Interface, Collaborative Playing, Game.

\section{Introduction}

Virtual heritage environment is the recreation of cultural heritage using virtual reality technology which allows visitors to experience reconstructing a historical site that no longer exist or travel back in time to experience historical events [1]. In the past, however, most virtual heritage environments are designed for digital preservation focusing on the accurate restoring it to original appearance [2]. Hence, user interactions are typically navigation and passive observation of events in the virtual environment. Recently, interactivity has come into attention in the design of virtual heritage applications. For example, the intelligent tour guide agents, game design approach, and multimodal user interfaces were used to increase a user's cultural learning experience and give innovative ways of storytelling behind the scene $[4,5,6$, 7 , 10]. Also, tangible user interfaces are used in the educational applications to strengthen the engagement of children in learning activities [9].

In this paper, we present the Tangible Moyangsung system, a virtual heritage environment designed for a group of users to collaboratively play games using 
tangible interfaces to learn cultural background story of a Korean war-defensive castle, Moyangsung. According to local folklore, this castle was constructed by women. There is a traditional festival held annually on September $9^{\text {th }}$ of the lunar calendar which has been in place for many generations. In this festival, the Dapsungnori ceremony is a highlight event. The ceremony entails women to put stones on top of their heads and parade around the Moyangsung castle where they would leave the stones inside of the castle after the parade. Folklore says anyone who goes around the castle three times can go to the heaven.

The Tangible Moyangsung system consists of three main subsystems: the context server, the virtual environment, and the tangible interfaces. In this system, the tangible interfaces are provided to give a transparency of user interaction with the virtual environment [3]. The tangible interfaces include the Matdol interface (inspired by a Korean traditional stone-made hand mill), the touch-screen interactive map, and the 100-cell based LED board interface for user navigation and interaction with the virtual environment. The board interface allows multiple users to layer bricks by placing tangible transparent acrylic blocks. It is designed to help users engage in the activity which would increase group social interaction and the overall collaborative learning experience.

This paper describes an overview of design and implementation of the Tangible Moyangsung system. It will then discuss the results of a study conducted on six different groups to evaluate whether the Tangible Moyangsung system would bring a group of participants together to complete a given task more efficiently. The comparative study results between collaborative conditions versus individual condition showed that the participants put a lot more effort into collaborative work setting using tangible interfaces. It helped the participants to work cooperatively to enhance group performance. This paper will conclude with a look into the possible direction for future research.

\section{Tangible Moyangsung}

Moyangsung is located in Gochang-gun, Jeollabok-do, South Korea. It is also known as Gochang-Eupsung. The name 'Moyang' originates from the ancient geographical name of Gochang in Baekje, one of the three Kingdoms in ancient Korea. Unlike other castles in the same era which were constructed on plain fields as homes for people, Moyangsung was constructed on a hill as a fortress. It also consists of many 'Ongsung', a small castle built outside of the main gate to protect against possible enemy attack.

Fig. 1 shows the photograph of Tangible Moyangsung demonstrated at the X-edu studio at Jeonju University, S. Korea. In Tangible Moyangsung, players are first introduced to the historical background of Moyangsung and are given a mission. The mission of the game is to find and repair the damaged walls completely within tenminute period. The task involves the players to walk around the castle and search for the destroyed parts of the wall. Once they find the damages, they can repair the wall by putting the tangible blocks (i.e., Tetris-pattern blocks made of transparent acrylic plastic) on the pattern appeared on the board interface. As a reward for successfully completing the mission, the players receive an opportunity to experience 


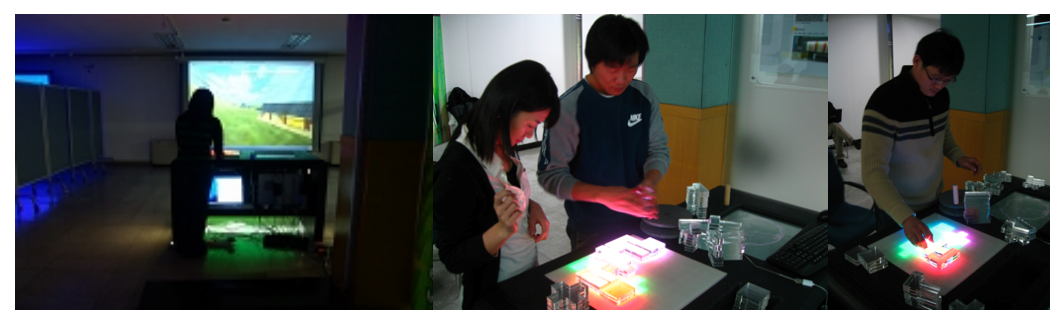

Fig. 1. The snapshot of users playing an easy (colored-pattern) puzzle on Tangible Moyangsung, installed at the $\mathrm{X}$-edu studio, at Jeonju University, Korea

Dapsungnori. When the players fail the mission, the castle will be destroyed completely.

In Tangible Moyangsung, the game starts with the introduction animation, and then the state is changed to the navigation mode. In the navigation mode, the players walk through the virtual environment using the Matdol interface or the touch-screen interactive map. When the players are close to the collapsed part of castle wall, the state is changed to the repair mode. Then, the same damaged pattern shown in the virtual environment is also displayed on the board interface in color lights. In the repair mode, the players place various shaped blocks on the puzzle pattern shown on the board interface. The state of user interaction with the board interface is periodically checked, and transparently updated in the virtual environment to display bricks filled the broken walls through message passing by the context server. The repair mode ends when the players complete the block placement correctly matched with the pattern, and the state is returned to the navigation mode. Finally, the game ends with the success when the players complete repairing destroyed walls in four different regions in the virtual environment within 10-minutes period. Otherwise, the game will be timed out - i.e., the game is over with the failure.

\section{System Design}

Fig. 2 shows the overall architecture of the Tangible Moyangsung system that displays the event and messages flows among the sub-components of the system. It consists of the context server for managing the main game logic, the virtual environment, and the tangible interfaces for user navigation and interaction. The context server includes the communication manager for the event and message handling among the sub-systems. The virtual environment shows the reconstructed Moyangsung (such as a castle, hills, trees, and buildings) with vivid sounds. It also contains user interaction and event processing modules for state update by the context server and the tangible interfaces.

The tangible interfaces include the Matdol interface and the interactive map for navigation and the board interface for user interaction in the virtual environment. The Matdol interface allows users to rotate its handle to walk through the pre-defined path along with the perimeter of the virtual Moyangsung. The touch-screen interactive map shows the top-down view of the castle and the user's current location in the virtual 


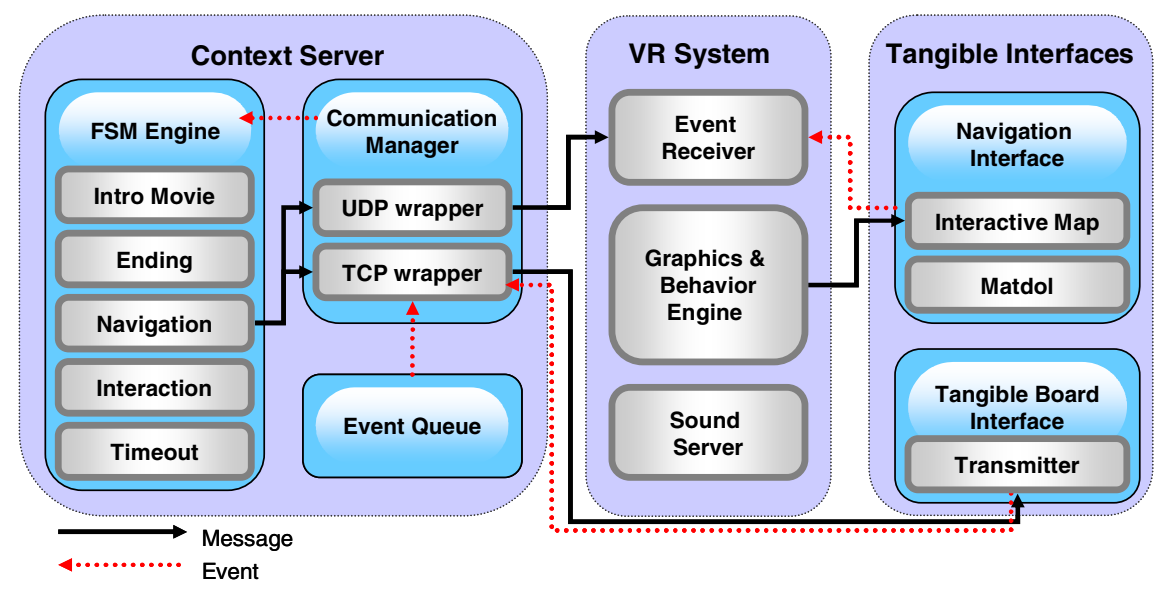

Fig. 2. The overall system architecture showing event and message flows among the context server, the virtual environment, and the tangible interfaces

environment. It allows a user to draw a path on the map with his/her finger to navigate in VR. The board interface adopts the Tetris puzzle game metaphor. It allows users to fortify the castle using various tangible blocks. Each block position is detected by 10x10 PIC micro-controller driven switch unit. Each micro-controller cell unit controls LEDs and switches. The board interface is connected to the communication manager by TCP.

\subsection{Context Server}

The context server maintains the game state from the beginning to the end as the players interact with the tangible interfaces in the virtual environment. As shown in Fig. 2, the context server consists of a finite state machine (FSM) controlling the various stages of the game logic and a communication manager employing an eventdriven message passing mechanism. The main game logic states are timer, game introduction and ending mode, user navigation mode, and user repairing mode. Events are generated from various sub-components; for example, the virtual environment sends the player's current position events to the context server for checking whether the players are close to the damaged castle region. In addition, when the players put the tangible blocks on the board interface during the repair mode; the board sends the events to the context server that are passed to the virtual environment to display the repairing progress.

In the context server, each module is implemented as an independent thread. The threaded event queue stores the events received from the sub-components through the communication interface. The communication manager has UDP and TCP module for processing the messages and the events. The communication with the board interface is mediated by the transmitter program, sending the messages through the RS485RS232 converter. The transmitter program also monitors all the cells on the board interface and sends the cell state report messages to the context server. 


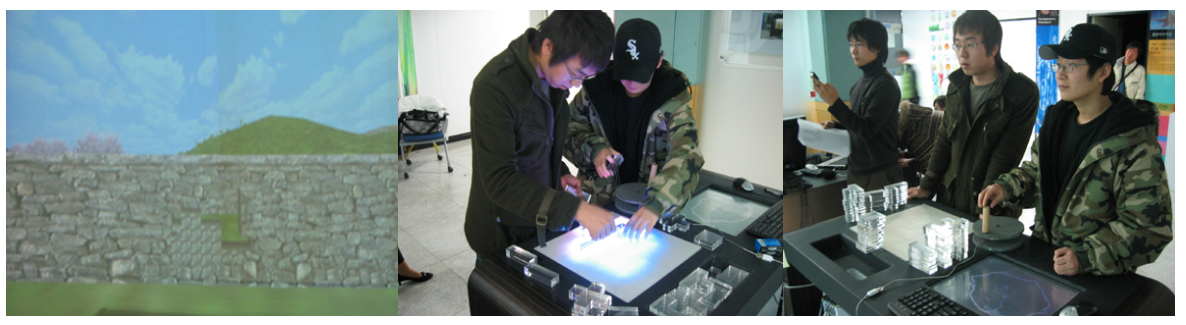

Fig. 3. On the left shows user repairing the damaged wall of the Moyangsung castle in the virtual environment; on the middle shows a pair solving a difficult puzzle (blue color) using tangible blocks; on the right shows a user navigating the virtual environment using the Matdol interface

\subsection{Virtual Environment}

In Fig. 3, the picture on far left shows the progress of user's repairing the Moyangsung damages in the virtual environment. The middle picture shows two users repairing the damaged wall (shown as the difficult puzzle pattern appeared on the board interface) using tangible blocks. The picture on far right shows a user navigating the virtual environment using the Matdol interface. As a new block is placed or removed from the board, a virtual stone is added or removed from the corresponding position of the destroyed wall in the virtual environment. This repair mode ends when the user finishes putting all the blocks in the right places on the board.

The environment is designed for users to participate in the tangible virtual reality experience. It contains the graphics modules (such as model loaders, animation, and navigation and manipulation interaction), the sound modules, and the event receiver module. It is written using the Ygdrasil, a high-level virtual reality authoring toolkit [5]. The current implementation of virtual Moyangsung installed at Jeonju University runs on a large-screen passive stereoscopic virtual reality system, built using the two low-cost DLP projectors, polarization filters and screen, and a Linux PC.

\subsection{Tangible Interfaces}

The tangible interfaces are the Matdol interface and the interactive map for user navigation and the tangible board interface for users when building bricks in the virtual environment. Matdol is a Korean traditional stone hand-mill. Matdol consists of two cylinder-shaped stones overlapped one on top of another, and there is a handle on the side of the upper stone which one can grind the grain using a Matdol by rounding its handle. In Tangible Moyangsung, users can move in the left or right direction along with the pre-defined path facing the exterior of the virtual Moyangsung by moving the Matdol interface as shown in Fig. 3 (on the right). The touch-screen interactive map displays the top-down view of Moyangsung and the user's current location in the virtual environment. Using the interactive map, a user can walk around the virtual environment by drawing a path on the map with their bare finger. 
The tangible board interface is designed to ease the virtual Moyangsung repairing. The board consists of $10 \times 10$ cells that have uniform squares $\left(40 \times 40 \mathrm{~mm}^{2}\right.$ for each cell). Users can play the puzzle with five different shapes of blocks (i.e., I, L, T, cross, and square shape). As shown in Fig. 3, the damage pattern is displayed on the board during the repair mode. Users' placement or removal of blocks on this board is reflected into the virtual wall. Tangible block turns on the switch embedded on each cell of the board. The LED lights on each cell present the multi-colored pattern to indicate the destroyed pattern. In addition, red, white, and black LED color is used to indicate the state whether the block placement is wrong, correct, or invalid. This tangible board interface is particularly designed for multiple users' simultaneous interaction with the virtual environment to encourage user engagement.

Each cell has its own PIC micro-controller; the controller drives the multi-colored LED lights and the switch turned by a block. Each cell controller has RS485 communication driver which is connected with a single RS485 bus. For each cell, a command can be sent to the controller to illuminate the appropriate LED color. If the board interface status is changed, the cell controller reports the changes to the context server, which then sends a message to change the light color or cell state on the board. As the game progresses, the board interface receives the cell check request message from the transmitter.

\section{A Case Study}

We conducted a case study of twelve persons divided into pairs using the Tangible Moyangsung system to evaluate how the system helped with group collaboration while playing the fortification game. Ten male and two female undergraduate students from Jeonju University volunteered as participants in this study. The range of the participants' age was 22 to 27 years old. They had moderate level computer experience, but most of them had no previous experience with the Tangible Moyangsung system. The game-play was a group to navigate the virtual environment and repair four destroyed areas of the castle. Repairing is done with the placement of all tangible blocks in the right places on the board.

\subsection{Method}

The experiment consisted of three playing conditions: (1) individual, (2) grouptogether, (3) group with role-division. In the individual condition, each group member worked alone, whereas the group performed the task together in the group-together condition. In the group with role-division condition, one member manipulated I- and cross-shaped blocks while the other manipulated L- and T-shaped blocks and then they shared square-shaped blocks. The participants were given two different levels of task difficulty: easy and hard. In the easy task, the block placement locations are indicated by five different colors (shown in Fig. 1), and the pattern is given by blue color only in the hard task (shown in Fig. 3).

The participants were first given an introduction of the system and the study overview. They were then asked to perform the task (both easy and hard task) under three playing conditions: individual, group-together, or group with role-division. 

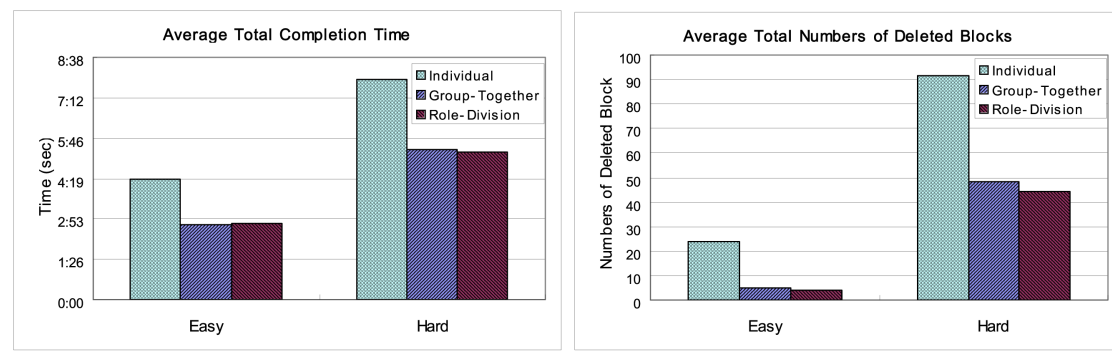

Fig. 4. The average total completion time (including navigation) and the average total number of delete blocks (indicating the extra number of trials)

Since the experiment was designed for a within-subject study, the order of condition was randomly given to minimize the participants' learning effect from previous experience with other conditions. Two groups performed the hard and easy task under the group-together condition, followed the role-division condition, and then the individual condition. The next two groups performed the easy and hard task under the individual condition, followed by the group-together condition, and then the roledivision condition. The reset of the groups performed the easy and hard task under the role-division condition, followed by the group-together condition, and then the individual condition. A post-test survey was followed at the end of the tasks. The surveys were intended to obtain user feedback regarding the overall system usability and the subjective evaluation on group collaboration and performance efficiency.

All groups in the various test conditions were recorded using a video camera. Their activities were also collected into log data files. These included the total completion time (from the game start to mission completion, including the navigation time), the block completion time for each damage repairing (from the block pattern emergence to the pattern completion), and the number of added or deleted blocks during the repairing mode.

\subsection{Results}

Fig. 4 shows the average total completion time (in minutes and seconds) and the average total number of deleted blocks for easy and hard task on three test conditions. The figure on the right (i.e., the averaged total number of deleted blocks) indicates the number of extra trials than the actual number of block appeared on the pattern. The pattern was easily distinguished by the five distinct LED color lights on the easy task while it was displayed by blue LED light on the hard task. As shown in Fig. 4, users performed significantly better under group-together or role-division than the individual play in both easy and hard task. However, there was no significant overall difference between the group-together and role-division condition although the number of deleted blocks was slightly reduced in the role-division condition.

Table 1 shows the average total completion time and the average total number of deleted blocks (as shown in Fig. 4). It also shows the average block completion time (in minute and seconds) and the average navigation time (in minute and seconds) for each condition. The navigation time was calculated by the difference from the average 
Table 1. Performance on individual play, group-together, and role-division condition

\begin{tabular}{|c|c|c|c|c|c|}
\hline Tasks & Conditions & $\begin{array}{l}\text { The average } \\
\text { total } \\
\text { completion } \\
\text { time }\end{array}$ & $\begin{array}{ll}\text { The average } \\
\text { total number } \\
\text { of deleted } \\
\text { blocks }\end{array}$ & $\begin{array}{l}\text { The average } \\
\text { block } \\
\text { completion } \\
\text { time }\end{array}$ & $\begin{array}{l}\text { The average } \\
\text { navigation } \\
\text { time }\end{array}$ \\
\hline \multirow{3}{*}{$\begin{array}{l}\text { Easy } \\
\text { task }\end{array}$} & Individual & $4 \mathrm{~min} 17 \mathrm{sec}$ & 24 & $48 \mathrm{sec}$ & $1 \mathrm{~min} 6 \mathrm{sec}$ \\
\hline & $\begin{array}{l}\text { Group- } \\
\text { together }\end{array}$ & $2 \min 41 \mathrm{sec}$ & 5 & $25 \mathrm{sec}$ & $1 \mathrm{~min} 2 \mathrm{sec}$ \\
\hline & $\begin{array}{l}\text { Role- } \\
\text { division }\end{array}$ & $2 \min 44 \mathrm{sec}$ & 4 & $28 \mathrm{sec}$ & $51 \mathrm{sec}$ \\
\hline \multirow{3}{*}{$\begin{array}{l}\text { Hard } \\
\text { task }\end{array}$} & Individual & $7 \mathrm{~min} 52 \mathrm{sec}$ & 91.5 & $1 \mathrm{~min} 34 \mathrm{sec}$ & $1 \mathrm{~min} 36 \mathrm{sec}$ \\
\hline & $\begin{array}{l}\text { Group- } \\
\text { together }\end{array}$ & $5 \min 20 \mathrm{sec}$ & 48.3 & $1 \mathrm{~min} 7 \mathrm{sec}$ & $54 \mathrm{sec}$ \\
\hline & $\begin{array}{l}\begin{array}{l}\text { Role- } \\
\text { division }\end{array} \\
\end{array}$ & $5 \min 16 s e c$ & 44.4 & $1 \mathrm{~min}$ & $1 \mathrm{~min} 15 \mathrm{sec}$ \\
\hline
\end{tabular}

total completion time to the average block completion time multiplying by four (blocks). The result shows that the navigation time was also slightly faster in the group-together or role-division condition than the individual condition.

We expected that performance on the easy task was not significantly different among three test conditions because we believed that the only hard task should require help from the other group partner. However, some participants had a hard time finishing the easy task during the individual condition. All group showed that the total completion time was faster when they worked collaboratively (either group-together or role-division) than individually, especially for the hard task.

We also expected that overall performance would be better on the role-division than the group-together condition since the role-division condition forced them to be more cooperative. In our previous experience with group work using Tangible Moyangsung, we found that typically only one group member put blocks while the other members just watched the progress. Sometimes the group members collided putting trying to put the same blocks on the same position on the board. However, unexpectedly in the current study, all group members eagerly collaborated to complete the task as fast as possible regardless of the group-together or role-division condition.

In the pos-test survey, most participants believed that collaborative work helped improve performance by sharing insights between group members. They said that task performance was the most inefficient on the hard task under the individual condition. However, interestingly enough, they insisted that they could concentrate on the task more easily during the individual condition. According to the post-test results, the participants said that they collided with other members the most often on the hard task under the group-together condition and the least often on the easy task under the roledivision condition. Interestingly, most participants strongly believed that the roledivision condition was better for group cooperation because it helped them increase discourses to complete the task. On the other hand, the participants could still work independently without a conversation since the group-together condition allowed anyone to put any blocks on the board. 
The video analysis showed the same result by the post-test survey. We found that the role-division condition (where one member allowed using I- and cross-shaped blocks while the other member allowed using L- and T-shaped blocks only) made the group members to discuss more often. In general they talked to his/her partner a lot to ask for putting the partner's blocks on the board. However, the users seemed to be hindered by this abrupt interrupt as well. Another interesting pattern we observed was that some participants (and groups) heavily utilized the I-shaped blocks to solve the puzzles instead of using other kinds of blocks, especially on the hard task.

\section{Discussions and Conclusions}

This paper presented a collaborative virtual heritage environment designed for a group to play games collaboratively using the tangible interfaces to share cultural experience. While most of virtual heritage applications focus on the reconstruction of cultural objects or places, this system is designed to encourage social interaction among group members which helps them to be more engaged in the cooperative activities.

The user study results showed that all groups performed significantly better during the collaborative condition (either the group-together condition or the role-division condition) since cooperation helped them see the same problem in various perspectives. In addition, when the users played individually, they seemed to be exhausted if the duration time got longer by solving the difficult puzzles. On contrast, the users did not show boredom or fatigue while they played collaboratively. Hence, even though the participants thought that the individual condition was better for concentrating on the task, the result revealed that one's power of concentration was indeed better during the collaborative condition than the individual condition.

In the future, we will also add an intelligent agent to guide users and modify the game story to add more interactive narratives [8]. For example, the tangible board pattern can be adaptively changed according to users' levels, and the scenario or educational contents can be rearranged by user's interests.

\section{References}

1. Gaitatzes, A., Christopoulos, D., Voulgari, A., Roussou, M.: Hellenic Cultural Heritage through Immersive Virtual Archaeology. In: Proc. 6th International Conference on Virtual Systems and Multimedia, Ogaki, Japan, October 3-6, 2000, pp.57-64 (2000)

2. Ikeuchi, K., Nakazawa, A., Hasegawa, K., Ohisi, T.: The Great Buddha Project: Modeling Cultural Heritage for VR Systems through Observation, In: Proc. of IEEE and ACM International Symposium on Mixed Reality and Augmented Reality (ISMAR'03)

3. McNerney, T.: Tangible programming bricks: An approach to making programming accessible to everyone. Masters Thesis, Media Lab, Massachusetts Institute of Technology, Cambridge, MA (1999)

4. Lee, Y., Oh, S., Woo, W.: A Context-Based Storytelling with a Responsive Multimedia System, In: Proc. of Virtual Storytelling 2005, pp.12-21 (2005)

5. Park, K., Leigh, J., Johnson, A.: How Humanities Students Cope with the Technologies of Virtual Harlem, Works and Days 37/38, 19 (1\&2), 79-97 (2001) 
6. Pape, D., Anstey, J., Carter, B., Leigh, J., Roussou, M., Portlock, T.: Virtual Heritage at iGrid 2000, In: Proc. of INET 2001, Stockholm, Sweden, 5-8 June 2001 (2001)

7. Song, M., Elias, T., Martinovic, I., Mueller-Wittig, W., Chan, T.: 3-2 VRC in edutainment: Digital heritage application as an edutainment tool. In: Procs of the 2004 ACM SIGGRAPH international conference on Virtual Reality continuum and its applications in industry

8. Steiner, K. E., Tomkins, J.: Virtual environments \& stories: Narrative event adaptation in virtual environments. In: Proceedings of the 9th international conference on intelligent user interface (2004)

9. Terrenghi, L., Kranz, M., Holleis, P., Schmidt, A.: A cube to learn: a tangible user interface for the design of a learning appliance. Personal and Ubiquitous Computing 10(2) (2006)

10. Watkins, J., Russo, A.: Digital Cultural Communication: Designing Co-Creative New Media Environments. In: Proc. of the 5th conference on Creativity \& cognition C\&C '05 (2005) 